\title{
Blindness resulting from orbital complications of ophthalmic zoster
}

\author{
Anna Moniuszko', Magdalena Sosnowska², Agata Zajkowska², Adam Garkowski², Piotr Czupryna ${ }^{1}$, \\ Sławomir Pancewicz ${ }^{1}$, Joanna Zajkowska ${ }^{1}$
}

${ }^{1}$ Department of Infectious Diseases and Neuroinfections, Medical University of Bialystok, Bialystok, Poland Head of the Department: Prof. Sławomir Pancewicz MD, PhD

${ }^{2}$ Student Association at the Department of Infectious Diseases and Neuroinfections, Medical University of Bialystok, Bialystok, Poland Head of the Department: Prof. Sławomir Pancewicz MD, PhD

Postep Derm Alergol 2015; XXXII (5): 396-399

DOI: $10.5114 /$ pdia.2015.48041

\begin{abstract}
Herpes zoster ophthalmicus occurs when the latent varicella zoster virus (VZV) reactivates in the trigeminal ganglion and ophthalmic branch of the trigeminal nerve. In the elderly, there is a sharp increase in the tendency of secondary skin bacterial infections occurrence due to the deterioration of capabilities of self-care and changed sanitation. We present a case of patient who developed phlegmon of the orbit, which resulted with complete unilateral blindness. Varicella zoster virus infection in the elderly may have a severe course due to the progressive weakening of the immune system related to the age. Moreover, skin lesions around the eye socket require special care in prevention of bacterial superinfections due to the extremely high risk of life-threatening complications or disability. Neuralgia resistant to pharmacological treatment present in the course of ophthalmic zoster and difficulty in caring about skin lesions predispose to the occurrence of complications.
\end{abstract}

Key words: herpes zoster ophthalmicus, varicella zoster virus, phlegmon of the orbit.

\section{Introduction}

Herpes zoster ophthalmicus occurs when the latent virus responsible for chickenpox and herpes zoster development (varicella-zoster virus - VZV) reactivates in the trigeminal ganglion and ophthalmic branch of the trigeminal nerve. It has been estimated that herpes zoster incidence is diagnosed in 4-5 per 1000 patients, from which $10-20 \%$ cases are herpes zoster ophthalmicus. Dysfunction of the immune system progressing with age, especially the cellular response impairment, and concomitant with other diseases, is strongly associated with a significant increase in the risk of a severe and complicated course of the VZV infection [1]. Neurological and ophthalmological complications, which are a result of virus activation per se, are present the most often. The other complications such as scarred lid malfunction or loss, paralytic ptosis, conjunctivitis, episcleritis, scleritis, infectious or neurotrophic keratitis, iridocyclitis, haemorrhagic retinitis, acute retinal necrosis, choroiditis, papillitis, retrobulbar neuritis, optic atrophy, Argyll Robertson pupil, pupillary paralysis, internuclear ophthalmoplegia, acute and chronic glaucoma, orbital apex syndrome and sympathetic ophthalmia, may occur in the V1 nerve distribution of the trigeminal nerve. All of these complications may lead to blindness [2].

In the elderly, there is a sharp increase in the tendency of secondary skin bacterial infections occurrence due to the deterioration of capabilities of self-care and changed sanitation. Improper hygienic care within the lesions, which are the main symptoms of herpes zoster, and neuralgia which is resistant to pharmacological treatment are considered to be factors increasing the risk of bacterial superinfection of pathologically changed skin areas [1, 3].

Orbital phlegmon is a rare, life-threatening inflammation of orbital soft tissue occurring with a greater frequency in children than in adults. Usually it develops as a consequence of the paranasal sinuses inflammation spread. Sporadically, the preseptal soft tissues inflammation can occupy the intraorbital space through anatomical continuity. The most common complications of the orbital soft tissue inflammation are subperiosteal abscesses and intraorbital abscesses. Much less frequently the infection may spread into the cranial cavity and lead

Address for correspondence: Anna Moniuszko MD, Department of Infectious Diseases and Neuroinfections, Medical University of Bialystok, 14 Zurawia St, 15-540 Bialystok, Poland, phone: +48 85740 95 14, fax: +48 85740 95 15, e-mail: annamoniuszko@op.pl Received: 22.02.2014, accepted: 17.07.2014. 
to permanent loss of vision. However, in most cases appropriate therapeutic and diagnostic procedures enable avoidance of complications [4-6].

In this paper, we report a case of permanent, unilateral blindness occurring as a complication of bacterial inflammation of the orbital soft tissues in the course of herpes zoster covering the I branch of the trigeminal nerve in a 85-year-old patient.

A 85-year-old Caucasian female was admitted to the Department due to a suspected ocular herpes zoster lasting for 2 weeks before hospitalization. In anamnesis she reported hypertension and cholecystectomy. On admission the erythema and oedema of the left eyelids and the forehead region with numerous erosions were seen (Figure 1). The ophthalmological examination result on admission showed no signs of keratitis. The occurrence of described lesions was preceded by small grouped blisters on the erythematous surface and by general weakness and malaise. The patient was treated with oral acyclovir for 7 days. The eye-drops of amikacin were applied to the conjunctival sac. The lesions were treated with warm compresses, liquid powder with benzocaine and swabs soaked with Octenisept. The blood count performed during the hospitalization demonstrated features of megaloblastic anaemia (red blood count (RBC) $2.55 \times 10^{12} /$, haemoglobin (HGB) - $10.1 \mathrm{~g} / \mathrm{dl}$, haematocrit (HCT) 29.5\%, mean corpuscular volume (MCV) $115.7 \mathrm{fl}$ ). Thus, gastroscopy was performed, which revealed gastritis. Severe pain in the area of the ophthalmic nerve reported by the patient was treated with paracetamol - $500 \mathrm{mg}$ orally 3 times daily, tramadol - $100 \mathrm{mg}$ orally 3 times daily and carbamazepine $-200 \mathrm{mg}$ orally 2 times daily. Although the

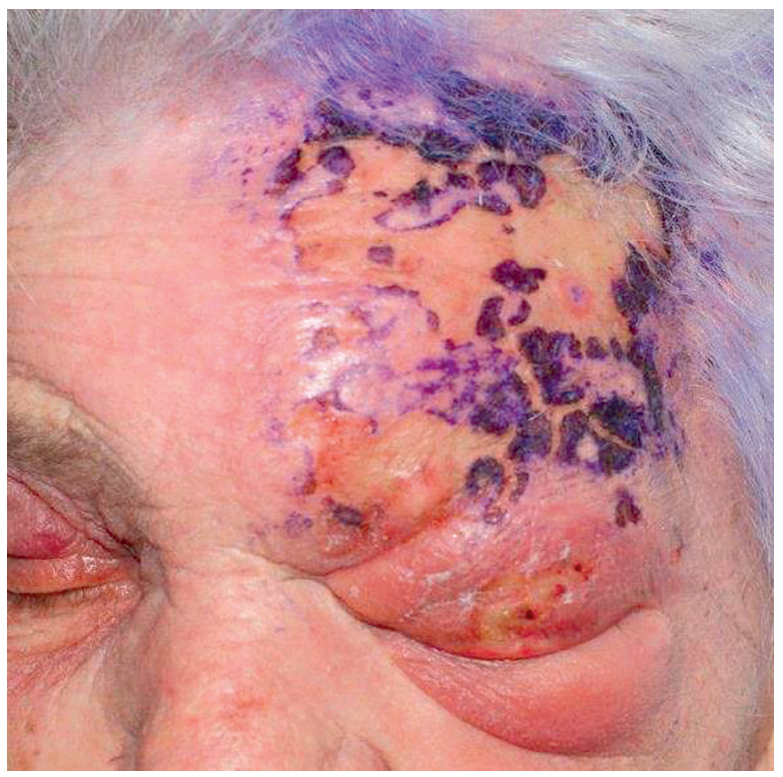

Figure 1. The erythema and oedema of the left eyelids and forehead region with numerous erosions doctor recommendations were different, due to the significant pain increase, the patient put compresses from non-sterile items in the vicinity of skin eruptions. Furthermore, the additional wound contamination occurred as a result of constant scratching of the diseased skin by the patient. On the $11^{\text {th }}$ day of hospitalization, lesions around the left eye intensified and a hard, sore swelling of the left eyelids limiting the eye mobility. The patient had no fever appeared. The laboratory studies showed elevated values of white blood count (WBC) $17.23 \times 10^{9} /$, C-reactive protein (CRP) $163 \mathrm{mg} / \mathrm{l}$, fibrinogen - $816 \mathrm{mg} /$ $\mathrm{dl}$ and D-dimer - $2.13 \mathrm{mg} / \mathrm{l}$. The patient was consulted by specialists in ophthalmology and otolaryngology. In the ophthalmic examination a hard swelling of left eyelids, as well as misaligned stare with side-downwards left eyeball rotation, and conjunctival swelling and hyperaemia, was observed. Moreover, visual acuity - no light-sensing, a significant reduction in mobility and irregular pupillary dilation of the left eye was described. The otolaryngological examination excluded sinusitis as the cause of intraorbital complications. A head computed tomography (CT) with a contrast agent was performed, which revealed forward displacement of the left eyeball, elevated density of adipose tissue surrounding the left eyeball and the elongation with simultaneous thinning of the left optic nerve (Figure 2). Left orbital bone structures remained unchanged. However, there was no growth of microorganisms in blood culture, the culture swabs from the conjunctiva of the left eye grown the methicillin-resistant strain of Staphylococcus aureus (MRSA). The patient was initially treated empirically with antibiotics - vancomycin (1000 mg intravenously 2 times daily), ceftriaxone (2000 mg intravenously 2 times daily)

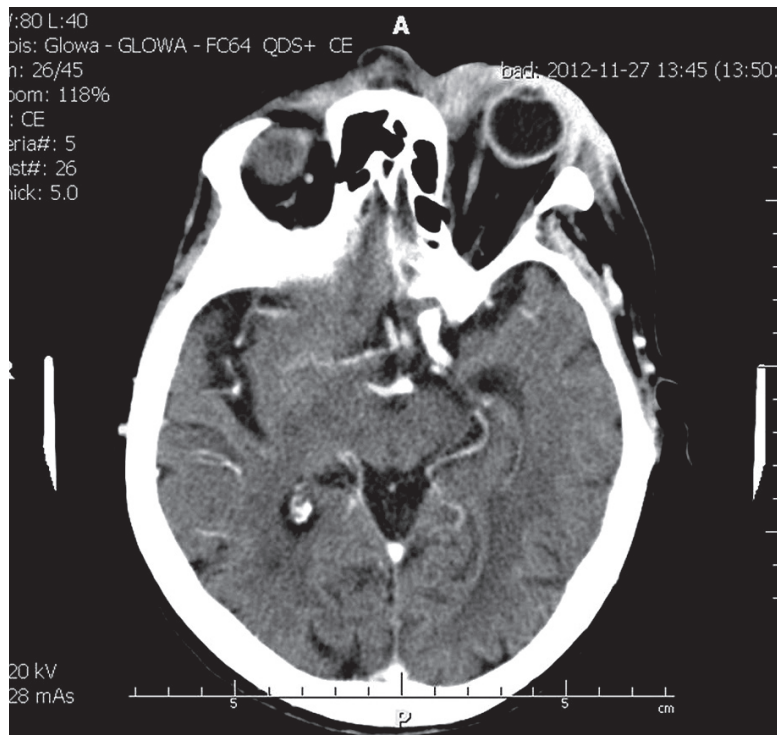

Figure 2. Displacement of the left eyeball, elevated density of adipose tissue surrounding the left eyeball and the elongation with simultaneous thinning of the left optic nerve 


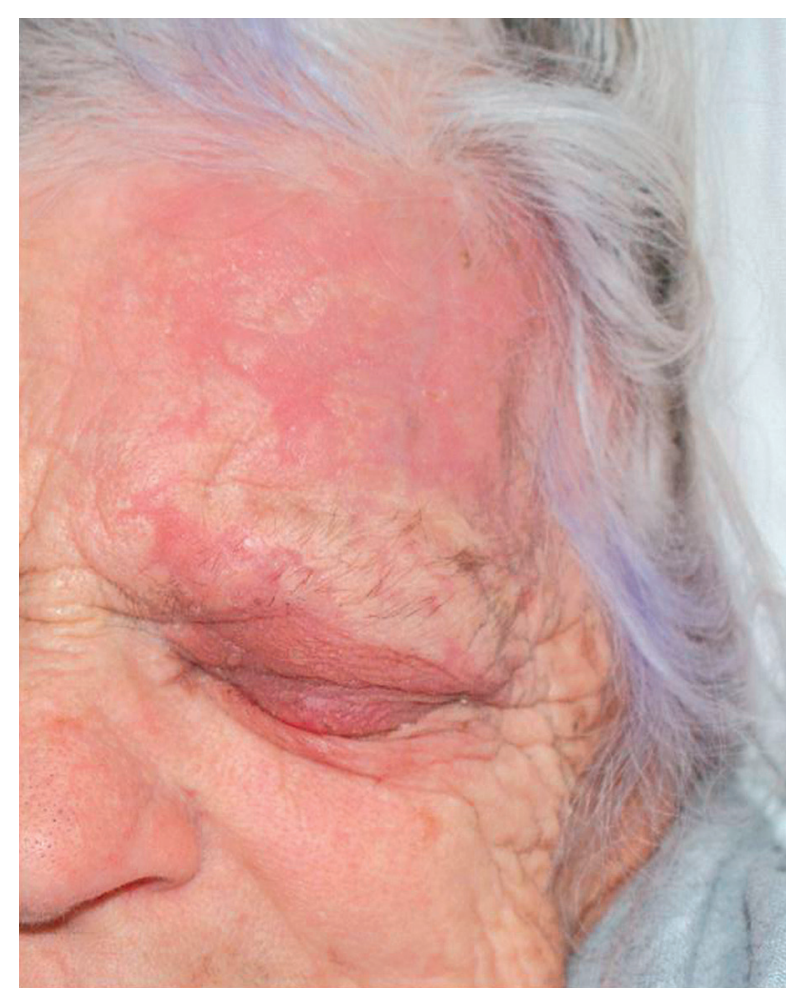

Figure 3. Healed skin lesions in the front temporal area and left eyelids

and metronidazole (500 mg intravenously 3 times daily). Five times daily drops with amikacin were used to the conjunctival sac of the left eye. After receiving the result of the inoculation of the left eye conjunctival swab, the administration of vancomycin was continued at a dose of $1000 \mathrm{mg}$ intravenously 2 times daily for 6 days, and additionally linezolid was given at a dose of $600 \mathrm{mg}$ intravenously 2 times daily for 12 days in accordance with the antibiogram. Then trimethoprim-sulfamethoxazole was given to the patient at a dose of $960 \mathrm{mg}$ orally 2 times daily. As a result of chosen therapy a clinical improvement, healing of skin lesions and reduction of oedema of the eyelids was achieved (Figure 3). However, the vision in the left eye did not return.

After 29 days of hospitalization, the patient discharged herself from the hospital at her own request. The continuation of the use of oral antibiotics for 2 weeks, and the control at the Ophthalmology Outpatient Clinic, Gastrological Outpatient Clinic and Pain Treatment Outpatient Clinic were recommended.

Both acute and chronic inflammation of the paranasal sinuses, especially ethmoid, is responsible for over $98 \%$ of cases of the bacterial soft tissues inflammation of the orbit. Another, much less frequent causative agents are orbital penetrating injuries, the spread of other local inflammatory processes and the complications of surgical procedures. There is also a possibility of spread of the infection through blood $[7,8]$. In the presented case as a result of a secondary bacterial infection of herpes zoster lesions, spread of the inflammatory process from the eyelids through anatomical continuity inside the eye socket occurred.

The transmission of MRSA requires contact with a colonized person or use of infected objects or materials [9]. The contamination with methicillin-resistant Staphylococcus aureus strain occurred as a result of the use of non-sterile compresses and constant scratching of the lesions around the orbit by the patient. Characteristic factors forming the orbital phlegmon are usually bacteria that colonize the paranasal sinuses such as streptococci, staphylococci, Haemophilus influenzae and anaerobic bacteria [10]. In adults, in most cases mixed bacterial flora is isolated [8]. For infections preceded by the injury of the skin barrier it is Staphylococcus aureus that is considered to be the most common pathogen [9]. In the reported case, the secondary bacterial infection of skin lesions was caused by MRSA.

Thrombophlebitis of the orbit, occlusion of the retinal artery caused by increased intraorbital pressure, inflammation or direct compression of the optic nerve are the mechanisms that can lead to permanent loss of vision in 3 to $11 \%$ of patients diagnosed with orbital phlegmon $[7,11]$.

In the differential diagnosis of the described case, we primarily included the preseptal inflammation of the orbit in the form of the inflammation limited to the eyelids tissue. Conjunctival oedema, exophthalmos, limitation or lack of movement of the eyeball and optic nerve dysfunction are clinical signs suggesting the presence of the infection inside the eye socket. During the course of this condition, also fever, malaise and pain occur [12]. Presence of meningeal signs is an indication for lumbar puncture and cerebrospinal fluid examination [13].

The head CT scan with a contrast agent in the frontal and transverse planes, particularly focused on the orbits and paranasal sinuses, is an imaging method which makes differential diagnosis possible, assesses an accurate bone structure and qualifies for surgery. Magnetic resonance imaging in comparison with $\mathrm{CT}$ enables accurate assessment of intracranial complications and differentiation of inflammatory changes occurring on a preseptal and postseptal basis [14].

Herpes zoster should be treated in all, immunocompromised and non-immunocompromised, patients. Acyclovir, valacyclovir and famciclovir are recommended depending on renal sufficiency. These antiviral agents hasten the resolution of lesions, reduce the formation of new lesions, reduce viral shedding, and decrease the severity of acute pain. To reduce the pain associated with the infection nonsteroidal anti-inflammatory drugs or acetaminophen can be ordered in patients with mild pain. For more severe pain, especially in the course of postherpetic neuralgia opioids are recommended. In eye 
disease mydriatic eye drops to dilate the pupil and prevent scarring and glucocorticoids for keratitis should be administered. This combined treatment should result in prevention of complications [1].

The topical treatment of herpes zoster should include also cool to tepid wet compresses (if tolerated), antibiotic ophthalmic ointment administered twice daily (e.g., bacitracin-polymyxin), to protect the ocular surface; topical steroids (e.g., 0.125\% - 1\% prednisolone 2-6 times daily). No topical antivirals are recommended because they are ineffective [2].

The treatment of orbital phlegmon requires the cooperation of many specialists: ophthalmologist, otolaryngologist, and even a neurosurgeon. After diagnosis, immediate hospitalization is necessary and application of broad-spectrum intravenous antibiotics, which spectrum includes the most common pathogens causing inflammation of the orbit soft tissues. The recommendations concerning empiric antibiotic therapy advise administration of vancomycin intravenously at a dose of 30-60 mg per kg 2-3 times daily, with one of the following drugs: ceftriaxone - $2000 \mathrm{mg}$ intravenously once a day, and in the case of suspected cerebral complications, $2000 \mathrm{mg}$ twice a day or cefotaxime - 2000 mg intravenously 4 times daily, or ampicillin-sulbactam - 3000 mg intravenously 4 times daily, or piperacillin-tazobactam - 4500 mg intravenously 4 times daily. In patients with a suspicion of intracranial spread of the infection, vancomycin, ceftriaxone and cefotaxime also in combination with metronidazole - 500 mg orally or intravenously 3 times daily are additionally recommended in order to expand the spectrum of treatment against anaerobes. The targeted antibiotic therapy ought to be implemented after identifying the causative pathogen. According to the recommendations, the parenteral antibiotic therapy should last for 10-14 days, followed by a period of 23 weeks' oral antibiotic use. The administration of clindamycin is suggested at a dose of $300 \mathrm{mg} 3$ times daily in monotherapy or combination therapy: clindamycin or trimethoprim-sulfamethoxazole in combination with amoxicillin or amoxicillin-clavulanic acid. In the case of intracranial spread of the inflammatory process, organized abscess with a diameter greater than $10 \mathrm{~mm}$ and lack of response to 24-48 h of conservative treatment, the implementation of a surgical procedure is essential [5, 14-17].

Varicella-zoster virus infection in the elderly may have a severe course due to the progressive weakening of the immune system related to the age. Skin lesions around the eye socket require special care in prevention of bacterial superinfections due to an extremely high risk of life-threatening complications or disability. Neuralgia resistant to pharmacological treatment present in the course of ophthalmic zoster and difficulty in caring about skin lesions predispose to the occurrence of complications.

\section{Conflict of interest}

The authors declare no conflict of interest.

\section{References}

1. Cohen JI. Herpes zoster. N Engl J Med 2013; 369: 255-63.

2. Dworkin RH, Johnson RW, Breuer J, et al. Recommendations for the management of herpes zoster. Clin Infect Dis 2007; 44 Suppl 1: S1-26.

3. Liesegang TJ. Herpes zoster ophthalmicus: preventing ocular complications through vaccination Herpes zoster ophthalmicus. Ophthalmology 2008; 115: S3-12.

4. Soon VT. Pediatric subperiosteal orbital abscess secondary to acute sinusitis: a 5-year review. Am J Otolaryngol 2011; 32: 62-8.

5. Gappy C, Archer SM, Barza M. Orbital cellulitis. UpToDate 2013.

6. Kanski JJ. Clinical opthalmology. Elsevier Urban \& Partner, Wroclaw 2013.

7. Kloek CE, Rubin P. Role of inflammation in orbital cellulitis. Int Ophthalmol Clin 2006; 46: 57-68.

8. Nageswaran S, Woods CR, Benjamin DK Jr, et al. Orbital cellulitis in children. Pediatr Infect Dis J 2006; 25: 695-9.

9. Kaplan SL. Epidemiology and clinical spectrum of methicillin-resistant Staphylococcus aureus infections in children. UpToDate 2013.

10. Seltz LB, Smith J, Durairaj VD, et al. Microbiology and antibiotic management of orbital cellulitis. Pediatrics 2011; 127: e566-72.

11. Patt BS, Manning SC. Blindness resulting from orbital complications of sinusitis. Otolaryngol Head Neck Surg 1991; 104: 789-95.

12. Georgakopoulos CD, Eliopoulou MI, Stasinos S, et al. Periorbital and orbital cellulitis: a 10-year review of hospitalized children. Eur J Ophthalmol 2010; 20: 1066-72.

13. Szaflik J, Grabska-Liberek I, Izdebska J. Emergency in ophtalmology. PZWL, Warsaw 2004.

14. Buchanan MA, Muen W, Heinz P. Management of periorbital and orbital cellulitis. Paediatr Child Health 2012; 22: $72-7$.

15. Wierzbicka M, Balcerowiak A, Dąbrowski P. Orbital phlegmon in pregnant woman. Postępy w Chirurgii Głowy i Szyi 2012; 1: 11-4.

16. Bagheri A, Tavakoli M, Aletaha M, et al. Orbital and preseptal cellulitis: a 10-year survey of hospitalized patients in a tertiary eye hospital in Iran. Int Ophtalmol 2012; 32: 361-7.

17. Vairaktaris E, Moschos MM, Vassiliou S, et al. Orbital cellulitis, orbital subperiosteal and intraorbital abscess: report of three cases and review of the literature. J Craniomaxillofac Surg 2009; 37: 132-6. 